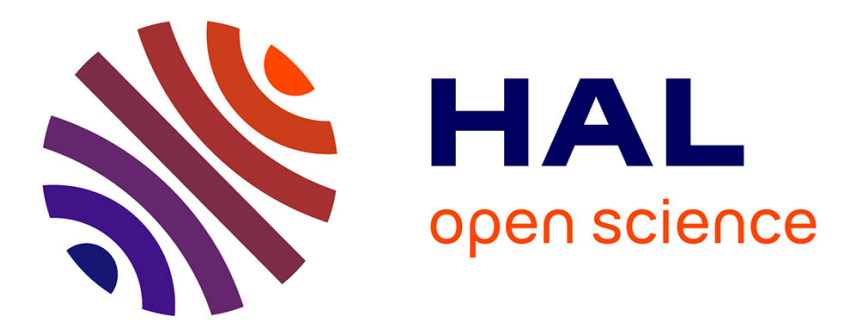

\title{
Trois utopies sonores pour la ville contemporaine
}

Olivier Balä̈

\section{To cite this version:}

Olivier Balaÿ. Trois utopies sonores pour la ville contemporaine. Espaces et sociétés (Paris, France), 2003, 115, pp. 60-77. hal-02161275

\section{HAL Id: hal-02161275 \\ https://hal.science/hal-02161275}

Submitted on 20 Jun 2019

HAL is a multi-disciplinary open access archive for the deposit and dissemination of scientific research documents, whether they are published or not. The documents may come from teaching and research institutions in France or abroad, or from public or private research centers.
L'archive ouverte pluridisciplinaire HAL, est destinée au dépôt et à la diffusion de documents scientifiques de niveau recherche, publiés ou non, émanant des établissements d'enseignement et de recherche français ou étrangers, des laboratoires publics ou privés.

\section{(1)(1) $\$(0)$}

Distributed under a Creative Commons Attribution - NonCommercial - ShareAlikel 4.0 


\section{Trois utopies sonores pour la ville contemporaine.}

Par Olivier Balaÿ.

Aujourd'hui, on entend dire que la société urbaine est bruyante. Elle a construit de grands ensembles d'habitations, des villes, des métropoles, des espaces de transport et de communication, et elle aurait échoué à aménager ces lieux pour l'ouïe. En ce début du $\mathrm{XXI}^{\mathrm{e}}$ siècle, on parle en effet du bruit nuisance, du bruit qui casse les oreilles. L'essentiel des expressions sur la ville sonore revient à censurer le bruit ou à le mesurer. Pourtant, à côté de ce discours dominant, d'autres formes d'expressions commencent à se faire entendre. On parle de plus en plus, dans les milieux de la recherche architecturale et urbaine, dans les bureaux de l'urbanisme opérationnel des grandes villes, de changer la qualité sonore urbaine ${ }^{1}$. Les bâtisseurs s'interrogent sur le paysage sonore de la ville, sur la sensibilité sociale aux phénomènes audibles et sur les rapports à faire renaître entre l'acoustique, l'architecture et l'urbanisme.

Or cette attention portée à la qualité de l'audible, qui s'est longtemps éclipsée du champ de la conscience culturelle des aménageurs, n'est pas nouvelle (Balaÿ, 2003.) Au début du XIX $\mathrm{XI}^{\mathrm{e}}$ siècle, avant l'essor de l'acoustique scientifique contemporaine, des utopies donnaient déjà des critères sur lesquels on pouvait fonder soit un confort acoustique, soit un univers sonore de remplacement à la ville traditionnelle. Porte-parole du désir d'un changement des rapports sociaux, leurs rédacteurs étaient à la recherche d'un idéal généreux, aspirant à des oasis de bonheur où se greffait souvent le rêve d'un grand changement et d'un dépaysement. Ils souhaitaient que l'ouïe soit à son aise et voyaient dans les constructions spatiales nouvelles un remède à la ville bruyante. D'un côté les visionnaires du début de l'ère urbaine industrielle pensaient que leur ville doit être un espace silencieux, calme et tranquille. A l'autre extrême, ils l'imaginaient comme un lieu sonore et animé.

En fait, le silence est un concept, ce n'est pas une réalité ; la littérature à travers de nombreux exemples montre que le silence est même plutôt négatif ${ }^{2}$. Par ailleurs, le bruit conçu comme une animation dense et riche n'est pas mesurable; c'est par l'écoute différentielle qu'il est vécu comme quelque chose de trop ou de pas assez vivace (Augoyard, 1978.)

1 Parmi d'autres, citons ces textes d'appels d'offres et de recherches récents suivants : «Construire avec les sons », PUCA 2000, «Le futur de l'habitat» PUCA 2002, Concevoir et réaliser: échelles et temporalités des projets urbains» PUCA 2002, «Qualité énergétique, environnementale et sanitaire : préparer le bâtiment à l'horizon 2010 » ADEME PUCA 2002.

${ }^{2}$ Cf. Balaÿ O. (2003), le chapitre intitulé «L'espace sonore dans les romans du XIXe siècle. » 
Nos utopistes sont donc confrontés à l'ambivalence de la notion de bruit: sa présence insupporte, mais son absence aussi. Ils rejettent la ville de l'époque en bloc parce qu'elle est cacophonique et bruyante, mais ils ne convoquent pas pour autant le silence total comme un cadre sonore idéal, parce que cela signifierait que la cité est paresseuse ou sans âme.

C'est là l'objet, très contemporain à nos yeux, de cet article historique : remettre en perspective le rôle du sonore chez les utopistes du $\mathrm{XIX}^{\mathrm{e}}$ siècle en repérant l'émergence des thèmes acoustiques qu'ils véhiculent, même si leurs aspirations produisent un discours clos sur les pratiques et les usages envisagés ${ }^{3}$. L'important, pour nous, c'est que ces idées sont exprimées en projets urbains précis et peuvent constituer des pistes de travail stimulantes pour l'époque actuelle dans la mesure où nous restons pleinement conscients de ne pas les transformer en justifications idéologiques.

Le second intérêt de ces textes tient au contexte de leur production. En ce début de l'ère industrielle, leurs rédacteurs ne peuvent pas se fonder sur des principes acoustiques fiables et quantifiés pour promouvoir le bienêtre sonore. Les connaissances scientifiques de l'époque ne sont en effet d'aucun secours pour les aménageurs, et ceci jusqu'au début du $\mathrm{XX}^{\mathrm{e}}$ siècle $^{4}$. Ceux qui se préoccupent de l'espace doivent plutôt croire au témoignage direct des sens et se tourner vers des choix éthiques, techniques, voir moraux, dont les finalités n'appartiennent pas directement à l'ordre d'un savoir scientifique. Ces textes appartiennent donc plutôt à l'ordre des savoir-faire acoustiques intuitifs qui ont rendu possible les démarches que nous allons découvrir. Or ces procédés ont été mésestimés jusqu'alors. Face à la technologie acoustique contemporaine, ils pourraient permettre de redonner une place à des démarches sonores créatives et renouveler les attitudes techniciennes dominantes.

\footnotetext{
${ }^{3}$ Leurs idées, comme l'indique Baczko (1978) dans son Lumière de l'Utopie, sont en effet des «combinaisons nouvelles de vieilles idées. »Ces images en retour leur auraient fourni un répertoire déjà tout constitué, qui explique en partie leur popularité : les références aux formes, signes, et valeurs historiques constituent, en effet, une des hypothèses explicatives de la sensibilisation de l'opinion publique au message utopique à l'époque. Cette hypothèse est reprise aussi par Ozouf M. (1984) et Zeldin T. (1979).

${ }^{4}$ Il faut savoir que l'intérêt que nos ancêtres ont porté au traitement phonique avant les années 1900-1930 ne fut pas d'ordre «scientifique », au sens entendu d'aujourd'hui. C'est en effet entre 1898 et 1915 que Wallace Clément Sabine, professeur de Mathématiques et de Philosophie à l'Université d'Harvard, jeta les fondements d'une nouvelle «science », l'acoustique architecturale, en découvrant la formule mathématique qui permet de calculer le temps de réverbération d'un local. Quant au sonomètre, d'abord destiné à mesurer la hauteur des sons, et non pas leur intensité, il ne sera vraiment utilisé dans ce dernier usage qu'à partir des années 1930 environ. Cf. Josserand P., Azais C. (1982), Astier L., Baron P., (1935).
} 


\section{Trois intuitions sonores du XIX ${ }^{\mathrm{e}}$ siècle.}

Dans quel contexte historique ces textes sont-ils rédigés ? Au début du $\mathrm{XIX}^{\mathrm{e}}$, les villes où croissait d'abord modérément la population vont subir des changements sonores qualitatifs profonds. Même s'il faut ne pas confondre urbanisation et industrialisation, les villes qui ont de nouvelles fonctions industrielles progressent en nombre d'habitants. Dans les plus grandes, cela se traduit par des surpopulations dans les quartiers du centre et des contrastes importants (Lequin, 1983) selon les lieux et les types de domiciliation (Chevalier, 1958.) L'étude des sources d'archives permet ainsi de saisir les réactions d'une autorité tracassée par le vacarme d'une vogue, par le bruit des hommes qui nettoient les fosses d'aisance, par les crieurs publics (Balaÿ 2003). Il en est de même de la délinquance bruyante, du parler fort, de la «déviance individuelle », comme le dit Yves Lequin. Ces «déviances », qui sont manières de répondre à une situation et à une déculturation urbaine nouvelle ${ }^{5}$, sont l'objet d'incompréhensions à peu près totales, et donc de censures.

Arlette Farge (1979) note, à propos de la capitale française, que « le logement et la rue se mêlent l'un l'autre sans qu'on sache exactement où commence l'espace privé et où finit l'espace public. » Et elle continue : «Le chevauchement des espaces et des fonctions est saisissant, les fenêtres guettent la rue ou l'interpellent, les ruelles mangent les maisons : tout ou presque se rejoint dans un même bruissement de vie où se préparent sans cohérence des lendemains agacés à travers un quotidien toujours difficile. » Berthelet de Barbot (1773) confirme ces impressions quand il écrit que la presqu'île de Lyon « est chargée de maisons séparées par des rues. » Aussi, parce que la maison n'est jamais close aux activités de travail, parce que les heures de la journée sont consacrées aux métiers et que ceux-ci n'immobilisent guère les compagnons, les gestes sonores et les voix qui rythment les heures et les conversations ne se séparent guère des activités de la rue. Pour ceux qui la vivent, cette circularité des sons entre la rue et l'appartement ne peut être vécue comme un emprisonnement. Mais pour ceux qui n'appartiennent pas à la rue, l'impression est toute autre.

Cette situation nouvelle de la population a éveillé les réflexions des réformateurs sociaux qui découvrent alors le renouvellement de

${ }^{5}$ Sur les processus de déculturation au XIX ${ }^{\mathrm{e}}$ siècle, Crubellier (1983) rappelle cette phrase de Jules Simon, un philanthrope ministre sous la troisième République : «L'ouvrier vit comme un paria, au milieu d'une civilisation inconnue et d'un courant d'idées qui ne descendent pas jusqu'à lui ; il n'est pour ainsi dire ni de son pays ni de son temps ; privé de toutes les joies intellectuelles, il se rejette, comme la brute, sur des plaisirs grossiers qui achèvent de le dégrader. » 
l'urbanisme comme un remède et englobent leurs propositions sonores dans des modèles d'organisation visuelle de l'espace. Or, ces conceptions optimistes de l'environnement acoustique sont comme des portraits de société. En relisant les pages de Mercier qui imagine en 1798 l'univers sonore de Montmartre à Paris en l'An 2440, la description des Salines de Ledoux (1804) et celle du phalanstère de Fourier (1829) nous avons saisi combien l'utopie se nourrit de la valorisation de la puissance de récollection des sons. Françoise Choay (1965) l'indiquait : nos deux derniers « utopistes » ont une connaissance approfondie des traités d'architecture et leurs œuvres se placent dans un moment où l'utopie « se mobilise pour tenter de dépasser son statut de livre et de passer à l'acte. " Le texte de Louis Sébastien Mercier se situe différemment quant à ces objectifs puisque, selon Baczko (1978), c'est un rêve sur des projets conçus pour la ville de Paris au XVIII ${ }^{\mathrm{e}}$ siècle. Ainsi, de ces textes qui impliquent plusieurs conceptions de l'espace sonore nous avons dégagé trois figures emblématiques que nous avons appelé réserve de silence, entretemps sonore et résonateur d'urbanité. Ces figures, on le verra, ne sont pas isolées. Elles se retrouvent, à d'autres époques et dans d'autres textes relatifs au contexte bâti : un texte littéraire de Georges Duhamel (1930) sur le paysage sonore campagnard idéal, un texte de Pline le Jeune (97-114) sur sa villa du Laurentin et des textes de l'architecte Le Corbusier (1923), notamment à propos de ses immeubles d'habitation conçus comme des « cités-jardins verticales ».

\section{Une réserve de silence dans Paris.}

Le tableau chimérique de Paris que dresse Louis-Sébastien Mercier, intitulé Montmartre en l'An 2440, est, selon Baczko, un rêve qui prend pour support spatial la ville telle qu'elle existe en l'An VII (1798). «J'entrai dans les carrefours spacieux où régnait un bon ordre... Je n'entendais aucun des cris confusément bizarres qui déchiraient jadis mon oreille... La ville avait un air animé, mais sans trouble et sans confusion (...). Tous les carrosses ont disparu, les appartements sont vastes et propres : quelle propreté, quelle fraîcheur en résulte dans l'air. » Partout des fontaines « laissent couler l'eau pure et transparente. »

La ville de 1798 est une ville bruyante. La foule y risque à chaque instant d'être écrasée par une innombrable profusion de voitures ; on « frissonne » dès qu'on entend les pas précipités d'une paire de chevaux qui avancent à toutes jambes parmi les femmes enceintes, les vieillards et les enfants ; comme c'est « insultant pour la nature humaine !» Ce point de vue est aussi bien proche de la pensée d'un Rousseau (1762) : quand 
Mercier écrit qu'il vaut mieux «aller chercher loin de ce Paris-prison, quelques villages d'un air pur et tranquille ", Rousseau s'exclame « Adieu donc Paris, ville célèbre, ville de bruits, de fumée et de boue... » Loin de cette cacophonie, on se dirige vers le Montmartre de l'An 2440 pour découvrir sur la colline un cadre de vie, calme: une réserve de silence, ombragée de toute part de «bois vénérables » et consacrée à la solitude. Une loi expresse y défend «qu'on frappe l'air aux environs d'aucun bruit discordant. » Plus loin, le guide de la colline idéale indique : «Vous avez vu sur votre chemin plusieurs de ces retraits isolés et charmants"; c'est là que se retire l'homme frappé du «pouvoir inconnu» qui lui commande d'écrire. Les académiciens sont des « chartreux. » Le calme sert au génie.

Ce miracle naît d'une entente : il n'y a pas d'intrusion sonore inopportune car les gens de la ville restent aux portes qui permettent d'accéder à la colline. Du point de vue de la morphologie urbaine, Montmartre ressemble donc à une zone de petits pavillons, "sorte d'ermitages où les savants et les artistes peuvent se livrer à la méditation, éloignés du bruit urbain. »(Baczko, 1978) Or, dans l'espace sonore réservé à ceux qui aiment les lettres et la culture, est aménagé un musée des bruits dans lequel tous les sons du monde sont conservés. Il est situé dans un pavillon à l'entrée duquel est inscrit « Abrégé de l'univers. » Le visiteur y découvre des pièces dans lesquelles tous les sons articulés de la voix humaine, mais aussi les cris des animaux, le chant varié des oiseaux, sont imités. Les bruits réels sont harmonisés, comme au théâtre, grâce au fonctionnement de «certains ressorts. » On y entend le rugissement des lions, des tigres et des ours. Naïvement, le chant des rossignols vient aussi « saisir » l'oreille qui perçoit jusqu'au « frémissement de leurs ailes amoureuses. » L'espace y joue un rôle qualitatif, à l'image de l'écho qui, «plus formidable encore » que dans les conditions naturelles, « répète le son au loin. »

On s'aperçoit très vite que ce «musée sonore » n'introduit pas seulement à la découverte des sons de la nature. Il sert aussi à convaincre les princes des abominations de la guerre. De ce musée des sons et de cette nouvelle manière d'entendre, Louis Sébastien Mercier attend en effet une nouvelle manière de comprendre le monde. Résumons : un jeune Prince, qui « inclinait à quelques passions belliqueuses », est conduit dans une salle nommée l'enfer : aussitôt, un machiniste met en jeu «les ressorts accoutumés », qui produisent à son oreille toutes les «horreurs d'une mêlée et les cris de la rage, ceux de la douleur et les clameurs plaintives des mourants, les sons de la terreur et les mugissements d'un affreux 
tonnerre, signal de la destruction, voix exécrable de la mort (...) Si la nature des bruits ne le soulevait pas alors dans son âme, s'il ne jetait pas un cri d'horreur, si son front demeurait calme et immobile, on l'enfermerait dans cette salle pour le reste des ses jours, mais chaque matin, on aurait soin de lui répéter ce morceau de musique, afin qu'il se contentât, du moins sans que l'humanité en souffrit. » La cacophonie sonore possède la vertu - ici toute pédagogique - de dégoûter le prince de ses passions guerrières.

Mercier vise en fait la juxtaposition de deux villes sonores dont les activités ne se mélangent pas. On trouve d'un coté une réserve de silence où tous les aspects sonores liés au déploiement incontrôlé et individuel $\mathrm{du}$ bruit sont absents et, de l'autre, une somme de territoires sonores emballés avec leurs signaux sonores respectifs. Mercier ne choisit pas entre l'une et l'autre ville, même si son cœur le pousse à préférer la plus silencieuse. Il retient les deux pour que les passions y trouvent leurs aises. Il se ferme à d'autres temporalités sonores. Il ne croit pas qu'on puisse se représenter l'environnement sonore urbain autrement qu'en opposant le bruit au silence. Ainsi, les flux sonores des deux parties ne pourraient plus alimenter l'écoute ordinaire. L'espace sonore idéal relèverait de la partition spatiale.

\section{Le parc de silence de Georges Duhamel.}

On retrouve encore cette anticipation visionnaire décrite dans un texte de Georges Duhamel qui date de 1930. L'auteur propose de découvrir sur la carte de la France une région négligée par les voies ferrées et les routes nationales mais, bien entendu, vivante, avec tous ses villages, ses coutumes, son humanité, son labeur : «un franc morceau de vingt kilomètres de côté. » Ce parc sera délimité par un enclos, et les voies d'accès seront surveillées. Il faut le rendre silencieux, «non pas ridiculement silencieux, mais raisonnablement, mais intelligemment silencieux », et le protéger, hors limites, par un périmètre de calme : « un silence de seconde zone. " À l'intérieur, pas de chemin de fer, pas de gare, sinon hors d'ouïe. Les autos du ravitaillement seront admises le matin, pendant une brève demi-heure, et conduites avec douceur. Pas d'autos le reste du jour : on pourra cheminer sur la route en devisant ou en rêvant dans une sécurité totale. Pas d'avion. Nul bruit industriel.

Enfin, point capital, nulle musique mécanique. Le silence : « un silence humain, sain, riche, et surveillé comme un objet de grand prix », rempli des bruits de l'établissement humain qui a pris place dans les lieux : les voix des «aïeuls, des enfants et des femmes », les activités de la maison, 
les rumeurs du travail et des "machines familières », qui se mêlent au « concert» des bruits du corps. A tous ces bruits familiers répondent, «mesure pour mesure », les bruits du hameau, ceux de l'environnement lointain, qui «se déroulent paisiblement à l'arrière plan de la connaissance. »

Ce parc de silence idéal est figé. En restaurant l'ancienne image sonore du village ou du bourg, Duhamel rend les sons inertes et l'auditeur passif. Il n'imagine pas l'établissement d'écoutes neuves ou non avenues. Dans son mouvement de re-personnalisation de l'environnement, des composantes sonores bien connues sont réunies en un environnement sonore synthétique, simplifié, que la délimitation du périmètre de calme permet de conserver. Cette idée de restaurer une harmonie sonore et un âge d'or situé dans le hameau d'antan a-t-elle beaucoup de sens ? Cette hypothèse d'un aménagement sonore opposant les zones de silence aux territoires bruyants peut-elle être reprise autrement? Claude Nicolas Ledoux y avait songé.

\section{Les entretemps sonores de la Saline de Chaux.}

Les écrits de Ledoux (1804) expriment un rejet total des formes de vies préindustrielles. Il n'envisage donc pas de chercher des solutions pouvant améliorer l'existence dans la ville en développement. Il n'envisage même pas de construire l'habitation à côté du lieu de travail des salines. Il décide plutôt d'espacer ces deux zones et de construire chacune en rapport avec son cadre naturel environnant. C'est pourquoi, loin des usines des Salines, est construit Arc-et-Senans.

Par rapport à Mercier, Ledoux éclaire pourtant le tableau sonore urbain utopique d'une façon différente, notamment dès qu'il aborde les conditions sonores des habitants. Il distingue pour chacun d'eux des temps d'activité différents, ce qui l'amène à mettre en correspondance l'activité de l'occupant avec les climats sonores de la demeure et ceux de l'extérieur, comme dans la maison de cet employé au moment du réveil, le matin : «Déjà, les zéphyrs sautaient de leur retraite, et la frâicheur du matin s'entendait dans la plaine. Déjà, la musique des airs se faisait entendre. L'oiseau précurseur du printemps éveillait le voyageur et propageait des sons harmonieux. » Quelques lignes avant, Ledoux laissait apparaître la préférence de ceux qui se laissent aller à de «célestes pensées » pour la tranquillité du soir : «On quitte tout, dit-il, on traverse l'onde épurative pour offrir à la divinité l'encens du culte le plus pur. »

Aucune demeure ne sera donc faite de façon semblable. Chacune sera construite pour rapprocher l'occupant des conditions sonores 
environnementales qui seront les plus favorables au ressourcement ou à la concentration au travail. Avec la demeure du mécanicien il avait le même souci : «La pièce principale, celle qui intéresse le plus l'artiste, est un cabinet retiré, loin des bruits inséparables des soins domestiques, à l'abri d'un caprice amoureux qui pourrait retarder, je dis plus, anéantir, l'inspiration du moment. Il faut l'éclairer par le haut afin que la pensée ne soit pas distraite par des objets extérieurs. Un arrière cabinet est nécessaire pour le préserver des importuns et des oisifs (...) $\mathrm{Si}$ l'architecte, sacrifiant son amour propre à la simplicité, à la commodité, à su procurer à la méditation du savant un asile impénétrable à la distraction, il aura bien mérité de la patrie, qui lui devra des découvertes utiles, soit au progrès des lumières, soit à l'aliment du savoir. »

Pénétrons dans d'autres maisons, celle-ci par exemple : «J'entre ; je trouve des chambres à coucher au midi (...) et le rez-de-chaussée occupé par des pièces destinées au service journalier. De petites cours révèlent tous les besoins de la vie. » Dans cette autre habitation, que nous dit encore Ledoux ? Qu'on ne peut y entendre " que le doux murmure des petites chutes et celui des cascades lointaines. »

L'architecte imagine en fait de rendre concordant les conditions d'écoute avec les temps d'activité et les espaces qu'il dispose pour édifier. Ces entretemps sonores ${ }^{6}$, où l'on entend le dedans et le dehors, plusieurs sources provenant des pièces d'habitation, jamais dans le même ordre, avec les paysages sonores modulés, filtrés ou distanciés qui y prennent place, sont l'objet d'une grande attention. Le méditatif est ainsi accompagné par les bruits de la nature dans un cabinet de travail où il ressent, le soir, l'agrandissement de l'espace en phase avec l'élancement de ses pensées. Pour celui qui se lève tôt le matin, l'environnement phonique a des plans d'écoute différents de manière à créer un sentiment de profondeur, entre les sons domestiques proches et les bruits naturels lointains. Enfin, à l'intérieur de la demeure du mécanicien, l'isolement phonique n'a de sens que s'il peut être à tout moment rompu.

Cette organisation s'affine dans la maison de Monsieur $\mathrm{M}^{* * *}$, où sont regroupées toutes les caractéristiques sonores de la douceur du foyer : mise à distance des sons proches, éloignement par rapport au fracas du monde, environnement de jardins propres à accueillir le calme le soir et les bruits de la nature le matin. Dans la « Maison du Directeur », Ledoux prend en compte les situations familiales essentiellement changeantes, comme le prouve le souci d'une distribution spatiale qui satisfait aux «possibilités de bien vivre, même entre membres qui ont des goûts

${ }^{6}$ C'est nous qui nommons 
opposés. » On y remarque la multiplication des petites pièces pour recevoir les papiers, les livres, les cabinets de travail. Il lui faut aussi réfléchir au traitement acoustique des espaces quand l'activité qui s'y déroule l'exige. Ainsi, la salle de réception du directeur où l'orateur, placé entre des murs dont les surfaces ont été « assourdies », parle depuis un autel annoncé de soixante marches qui montent de fond » : c'est là que le maître s'adresse à ses ouvriers. Il est donc dans la nature de l'architecte d'envisager l'écoute en rapport avec la disposition des pièces domestiques et l'humeur des occupants, de faire circuler les sons entre les espaces ou de qualifier les mouvances sonores.

Le même souci concerne l'espace public, visant à la fois l'éducation studieuse et la détente des membres de la ville de Chaux. C'est sous les voûtes de la Maison d'union, «fermées au centre, pour se garantir de la chaleur du midi, ouvertes au septentrion, pour rafraîchir l'air, que la foule qui se presse trouvera la salubrité et corroborera ses poumons ; c'est sous ces voûtes consacrées à la méditation, à la discussion des intérêts particuliers, à la science, à la collection des meilleurs livres, aux jeux qui occupent l'esprit, sans compromettre les mœurs, que l'on trouvera la réunion indépendante des caprices perturbateurs. »

Ledoux imagine encore un édifice destiné aux récréations, dans lequel le temps des activités est réglementé. Les activités en plein air mais à couvert, même les plus innocentes, sont inventoriées avec leurs bruits. Citons pêle-mêle : le bruit de croc-en-jambe d'un combat entre deux ouvriers, celui du forgeron qui chasse en lançant un bâton qui siffle dans les airs, citons encore les rires de jeunes femmes lors des jeux de pigeons, les acclamations des spectateurs au jeu de ballon, de paume, au jeu d'eau. On chante dans les cuisines, on joue de divers instruments de musique précise encore Ledoux. «Enfin, sur les huit heures, tout le monde se retire (...) et se promet de nouveaux plaisirs pour la féerie prochaine. » Ledoux est bien loin de voir nettement l'avenir de la cité industrielle moderne. (Ozouf, 1984) Son intuition d'une régénération de l'individu dans un environnement sensible maîtrisé par l'espace est enracinée dans son siècle. Sans doute, aussi, son point de vue négatif sur le bruit urbain est presque soupçonneux tant il insiste sur le comportement des sociétaires, allant jusqu'à placer dans leurs mains des objets sonores sensiblement moins bruyants que ceux qui sont utilisés dans la ville traditionnelle (la cornemuse plutôt que les cymbales, le boomerang à la place du fusil de chasse, l'outil plutôt que la machine. ) Mais sa démarche est originale parce qu'elle se donne comme exigence de construire des volumes intérieurs et des lieux publics dans lesquels les 
bruits circulent, rassemblent, et rendent possible la méditation studieuse comme la récupération de soi. L'univers sonore change. Les sons se déplacent, ils sont en mouvement, on n'entend jamais deux fois les mêmes sonorités.

\section{La pièce de distanciation sonore de Pline.}

Prenons le temps d'évoquer ici cette merveilleuse description d'un aménagement sonore dans le passé. Il s'agit d'une lettre que Pline Le Jeune adresse à Gallus à propos d'un pavillon de sa villa du Laurentin. On retrouve les traits d'un homme qui a besoin d'un paysage sonore approprié à ses plaisirs personnels, loin de la joie collective.

«A côté est une chambre pour la nuit et le sommeil. Ce lieu ne perçoit ni les voix des esclaves, ni le grondement de la mer, ni l'ébranlement des tempêtes, ni la lueur des éclairs, pas même la lumière du jour, sauf quand les fenêtres sont ouvertes. La profondeur de cette retraite et de cet isolement s'explique par l'existence d'un corridor entre le mur de la chambre et celui du jardin, aussi les bruits viennent-ils expirer dans le vide des parois. Contre cette chambre est une toute petite pièce de chauffage, ayant une bouche étroite par laquelle la chaleur venue d'en bas est réglée, tantôt déversée, tantôt retenue. Puis une antichambre et une chambre à coucher, s'avançant à la rencontre du soleil, l'accueillent dès son lever, et au delà de midi ne le conservent plus qu'avec des rayons obliques, mais enfin le conservent. Quand il m'a plu de me retirer dans ce pavillon, il me semble que je suis loin même de chez moi, et j'en goûte l'agrément surtout en temps de Saturnales, alors que tout le reste de l'habitation résonne des folies de ces journées et des cris de joie. Ainsi je ne gêne pas les plaisirs de mes gens et eux ne gênent pas mes études. » Pline parle-t-il de censurer le bruit, de rendre silencieux ses domestiques, même d'isolement ? Non ! L'auteur romain évoque les différentes temporalités de son habitation. Il décrit un dispositif qui annonce le rôle acoustique de l'antichambre dans l'habitation privée du XIXème siècle (Balaÿ, 2003). Il évoque le plaisir qu'il a de se retirer dans un endroit calme quand ses gens s'amusent. Cependant il insiste sur le fait que la retraite est un séjour temporaire, un refuge provisoire. Cela n'est pas durable. L'ouïe ne peut être à l'aise dans un milieu isolé du bruit social et naturel.

Ledoux avait compris cela. C'est pourquoi sa cité n'est pas paresseuse et à tendance à faire sonner les bruits de la nature, ceux du travail, ceux qui symbolisent l'utilité de se rassembler. Là on entend des conversations, là des rires, là un jeu, là un banquet, là le son d'une fontaine, comme s'il 
fallait absolument occuper l'ouie. Serait-ce le signe que l'espace entier doit résonner des sons de la sociabilité heureuse de vivre dans la cité idéale ? On peut le penser, et Charles Fourier partageait aussi cette intention.

\section{Le parcours résonateur de l'urbanité dans "La Phalange" de Fourier.}

L'auteur du Nouveau monde industriel et sociétaire a horreur de la cacophonie et ne se gène pas pour le dire. Ecoutons le dresser sa virulente critique d'un texte rédigé en 1817 par un autre utopiste, Robert Owen : «Les civilisés ayant communément l'instinct du faux ne manqueraient pas à préférer la plus vicieuse distribution. Cela est arrivé à New-Harmony, où le fondateur Owen a précisément choisi la forme de bâtiment qu'il fallait éviter, le carré ou monotonie parfaite. C'est jouer de malheur comme un milicien qui attrape le billet noir : l'un des inconvénients du carré est, que les réunions bruyantes et incommodes, les ouvriers au marteau, les apprentis de clarinette, seraient entendus de plus de la moitié du carré sur quelques points qu'on les plaçât. Je citerais vingt autres cas où la forme carrée causerait du désordre dans les relations (...) Du reste, son carré peut être bon pour des réunions monastiques, telles qu'il en fonde, la monotonie étant leur essence. » Fourier ne semble pourtant pas être arrivé à concevoir des espaces qui donnent une résonance acoustique juste à la vie sociale idéale qu'il imagine. Faisons en le constat.

Toute sa pensée sur l'audition va vers l'éducation musicale du sociétaire, vers l'isolement entre les zones de travail et celles qui assurent les différentes fonctions nécessaires à la vie collective. Rien donc de très nouveau par rapport à Ledoux, si ce n'est son obsession pour l'isolation phonique entre les espaces d'activité et entre les appartements. Il s'appuie pour cela sur une critique de la société sonore de son siècle : « Dans les ménages de famille, dit-il, l'enfant s'ennuie au point de hurler nuit et jour, sans que ni lui ni les parents puissent deviner les distractions dont il a besoin et qu'il trouverait dans un séristère de prime enfance. La morale veut que dans ce ménage de famille, le père se complaise à entendre le vacarme perpétuel de marmots, qui le prive de sommeil et trouble son travail. La nature veut au contraire que l'homme, pauvre comme riche, soit délivré de ce charivari moral et que, rendu à sa dignité, il puisse reléguer en un local éloigné cette diabolique engeance, placer les enfants en lieu où ils soient sainement et agréablement tenus, selon la méthode sociétaire qui assure le repos des pères, des mères et 
des enfants ». Ils sont tous harcelés, écrit encore Fourier, par le « régime civilisé nommé doux ménage, véritable enfer pour le peuple, quand il n'a ni appartement séparé pour les marmots, ni argent pour subvenir à leurs besoins. (...) La morale veut qu'on place autour de l'enfant une demidouzaine d'aïeules et tantes, sœurs et cousines, voisines et commères pour lui créer des fantaisies qui nuisent à sa santé, et lui fausser l'oreille par la musique française. La nature veut qu'on n'emploie pas le vingtième de cet attirail pour tenir l'enfant gaiement et sainement dans un séristère assorti à tous les instincts du premier âge ». C'est ainsi que les enfants seront cloisonnés dans les lieux et éloignés des espaces de repos des parents, que l'éducation de l'ouïe privilégiera la musique et l'opéra et que les zones d'artisanat, les marchés bruyants, seront séparés des autres parties de la ville.

Lorsqu'on passe en revue les dispositions concernant la sensibilité sonore, force est de constater que les plaintes, les cris, les chansons, les hurlements, tous les bruits ordinaires sont absents de la Phalange. L'important tient dans la pratique de la musique en groupe, dans les signaux sonores qui rappellent aux sociétaires l'organisation sociale du phalanstère. Le son des clairons, des tocsins, «sera là pour réunir » les individus.

Constatons donc que Fourier habite l'environnement sonore en «militaire. » Il se représente le bruit «d'une immense armée invincible de travailleurs résolus et triomphants » pour qui il faut projeter un urbanisme approprié aux tâches à leurs mesures. Il veut inventer le dispositif qui évitera « ces insupportables fracas de toute nature répandu au hasard dans tous les quartiers des villes civilisées, où l'enclume des forgerons, le marteau du ferblantier, le flageolet, la clarinette, le cor de chasse, conspirent contre les oreilles publiques avec les grincements de violon, le tintamarre des voitures, et tous ces charivaris discordants, cassants, déchirants ou assourdissants, qui font de presque tous les appartements des grandes villes, de véritables enfers, enfin et par dessus tout avec le féroce, l'inévitable, l'indomptable piano »(Choay, 1965).

Dans cette cité sonore sans mystère, on apprend bien vite que la rue galerie est la pièce la plus importante ; «ceux qui ont vu la galerie du Louvre au musée de Paris peuvent la considérer comme modèle d'une rue-galerie d'harmonie, qui sera de même parquetée et placée au premier étage, et dont les croisées pourront, comme celles des églises, être de forme haute, large et cintrée, pour éviter trois rangs de petites croisées (...) Les abris et passages couverts sont un agrément dont les rois mêmes sont dépourvus en civilisation ; en entrant dans leurs palais, on est 
exposé à la pluie, au froid ; en entrant dans la phalange, la moindre voiture passe des porches couverts aux porches fermés, et chauffés ainsi que les vestibules et escaliers. L'ensemble sera bâti en briques et en moellons. » On retrouve dans Le voyage en Icarie de Cabet (1840) un ordonnancement du cheminement presque identique à celui prévu par Fourier. Le même principe a servi aussi à la construction du familistère Godin de Guise (1887) où les coursives d'accès aux logements donnent, pour leur part, sur une immense cour intérieure couverte d'une verrière. S'agit-il d'un promenoir sonore idéal ?

Joigneaux, dans ses Souvenirs historiques, raconte que dans les galeries couvertes de l'Abbaye de Cîteaux, la communauté Fouriériste, visitée par Arthur Young, faisait « sonner » les rires des bandes joyeuses qui s'y amusaient. (Petitfils 1982) Mais ce sont là aussi «des vases clos» précise t-il. Ainsi, l'image voulue d'une réciprocité forte des rapports humains dans la communauté semble paradoxale avec l'acoustique des galeries fermées et couvertes. Et c'est bien ce phénomène qu'on écoute aujourd'hui dans les espaces de ce type, par exemple les grandes galeries commerçantes ou les cages d'escalier d'un grand ensemble de logements : l'acoustique renforce soit la perception de la densité humaine soit celle d'un grand vide spatial et social, et la réverbération fait œuvre de dissolution de l'écoute des bruits multiples. (Balaÿ, Chelkoff, 1985.) Le zoning et l'isolation acoustique ne peuvent pas être des idées fécondes. L'erreur de Fourier, c'est de croire que le support spatial peut être un remède à tous les maux et que l'urbanité sonore est faite d'une somme de territoires acoustiques distincts les uns des autres. Or, cette mauvaise appréciation n'est pas isolée, puis ce qu'on la retrouve, par exemple, dans la façon de penser de Le Corbusier (1923).

\section{Les dispositifs d'isolation sonore chez Le Corbusier.}

Comme Fourier, Le Corbusier porte un jugement réducteur sur l'environnement sonore de son temps. Il répudie le tracé des rues « étroites pleines de bruits » dans sa ville idéale. Une ville moderne, ditil, «vit de droite, pratiquement (égouts, canalisations, chaussées, circulations). La droite est saine aussi à l'âme des villes. La courbe est ruineuse, difficile et dangereuse ; elle paralyse. » Le Corbusier (1923) est contre la rue traditionnelle. Dans son architecture, les cafés, les lieux de repos, etc., ne sont plus « cette moisissure qui ronge les trottoirs. » Ils sont reportés sur les terrasses des toits ainsi que le commerce de luxe, dans des lieux «consacrés au repos parmi les plantations de fleurs et de verdure. » «Le problème est posé d'habiter en «cités-jardins 
verticales » (Le Corbusier, 1946), méthode qui permet de rassembler les villes sur elles-mêmes, de libérer le sol à l'entour des logis, et qui permet enfin aux hommes de la civilisation machiniste de marcher à pied dans la ville en employant à nouveau leurs jambes » écrit-il. Comme Fourier, il part du constat qu'il faut abolir "nos médiocres boîtes à loyer mal insonorisées » (Le Corbusier, 1946) car le travail moderne «exige le calme ». Dans ses Unités d'habitations, les appartements sont groupés par deux, imbriqués «tête-bêche » au long des corridors d'accès appelés "rues intérieures". Ils «sont isolés l'un de l'autre par des boîtes de plomb. » (Le Corbusier, 1946) Et il poursuit : «Nous voici, dorénavant, capables de vaincre l'un des plus grands antagonistes de la construction moderne: le bruit. Peu importe que les matériaux soient bons conducteurs! Ceci n'entre pas en jeu. Les méthodes absolument scientifiques d'insonorisation permettent d'arriver presque à l'absolu dans ce domaine avec l'emploi des matériaux sonores ou insonores, à volonté. »

Dans ses textes, Le Corbusier accorde au logement standard isolé une place importante et privilégiée. L'habitant, depuis les fenêtres, voit un espace extérieur largement ouvert, troué de vide et de verdure. Mais qu'entend-t-il ? Et dans la rue intérieure couverte des Unités d'habitation de Firminy et de Marseille, par exemple, rencontre-t-il des gens, entendt-il des discussions, des voix ou bien seulement des pas et des clés ? Il faut aller sur place pour se rendre compte du sentiment « de vase clos » dont parlait Joigneaux. C'était pourtant autre chose qui était visée ! Ces insuccès répétés posent alors une question : les espaces sonores sont-ils condamnés à être le résultat d'une planification qui isole des bruits et qui envisage des espaces communs sans une acoustique adaptée au contexte et à la rencontre?

\section{Pour une utopie sonore ordinaire et contemporaine.}

Des lectures qui précèdent se dégagent en effet de nombreuses questions pour l'aménagement contemporain. Aires de silence, interstices à entretemps sonores, espaces de circulation résonateurs d'urbanité : est-ce que les bâtisseurs et les urbanistes actuels s'intéresseraient encore à ces intuitions sonores du passé ? On peut le penser, même si les principes destinés aux utopies sociales du XIX ${ }^{\mathrm{e}}$ ne sont pas tous des modèles d'espace sonore pour le confort de l'existence.

Qu'apporte alors notre contribution pour ce faire ? Nous en dégagerons trois pistes. Tout d'abord, ces utopies ont une caractéristique commune : elles confrontent les tares sonores de la ville existante à l'image d'un 
univers agréable pour l'ouie hors zone urbaine. Elles ne font pas de propositions d'interventions ponctuelles, simples, capables d'améliorer l'univers sonore urbain quotidien, comme s'il était impossible de remplacer les conditions phoniques qui s'offrent au citadin. Faut-il supposer que nos prédécesseurs avaient pressenti la disqualification actuelle des sons de la ville ? La question est importante. On trouverait ainsi formulée au $\mathrm{XIX}^{\mathrm{e}}$ siècle, par ces discours schématiques et réducteurs, l'esquisse d'une réflexion sonore qui ne se définit que par la censure du bruit, le quadrillage du silence et de la clarté sonore. La pensée sonore des utopistes aurait construit cette fausse évidence qu'on retrouve dans le discours acoustique actuel, à savoir que l'ouïe ne peut être à l'aise que dans un milieu isolé du bruit social et industriel. Désamorcer cette fausse idée est de la responsabilité actuelle de l'ensemble des intervenants de la construction. L'isolement du logement vis-à-vis des bruits extérieurs n'est pas la garantie du confort acoustique en ville. Délimiter des aires de silence est une illusion. Ce sont plutôt des temps de silence ou de calme auxquels il faut penser. Ainsi, c'est plutôt dans un projet global d'aménagement des flux sonores provenant du tissu urbain et de l'habitat que les bruits apparaîtront aux habitants et aux élus comme un espace de reconquête à leur profit.

Dans ce sens, et ce sera notre seconde conclusion, l'attitude particulière de Ledoux peut servir de guide. Ces textes montrent qu'il a bien senti combien la société est essentiellement affectée par des sons particuliers, émouvants, et pas seulement les sons du travail et ceux de la nature. L'architecte d'Arc-et-Senans tente l'harmonisation des deux types de sources sonores qui selon lui affectent l'esprit humain : le son de la vie sociale et le son d'origine passionnelle. Dans son modèle, il a besoin de la passion des individus rassemblés dans la cité. Mais le bruit que la société peut faire à ces moments reconnus comme importants, lors des naissances et des mariages par exemple, n'est jamais dominant. Mieux, il doit être fondu dans l'ambiance sonore qui ressemble à l'Usonie que Franck Loyd Wright décrira en 1958 ou encore à "l'urbanisation ruralisée » de $\mathrm{Cerda}^{7}$. Au risque de paraphraser Deleuze (1973) quand il analyse comment une collection d'idées devient un système, la forme d'un urbanisme sonore ruralisé est peut-être inventée par Ledoux : c'est le retentissement des actions sonores individuelles et familiales corrigées par les caractères sonores dominants liés à l'activité productive et aux

${ }^{7} \mathrm{Cf}$. par exemple, le passage où Cerda assimile les balcons et les fenêtres de la maison à des " organes correspondants aux yeux et à l'ouïe "in Choay F. (1980) p. 290 et 304. 
sons de la nature. Ces sons de la production et de la nature donnent une enveloppe, une épaisseur, un contexte aux formes d'existences sonores particulières. Il apparaît ainsi que dans ce rêve d'un architecte «atypique » (Choay 1980), l'action sonore individuelle et le fond sonore de la cité au «travail » vont de pair. Ce qui compte, c'est que l'activité sonore individuelle ou du groupe ne marque pas trop de dissonance par rapport au son du collectif, du travail et de la nature. Ledoux, on le sait, avait horreur du silence puisqu'il installe des fontaines sur les chemins de Chaux. Aussi l'architecte d'Arc-et-Senans désigne-t-il comme règle générative de son aménagement sonore, la nécessaire valorisation d'un fond sonore qui reflète les traits des activités sonores d'une culture.

Son approche de l'aménagement sonore, qui semble encore vivace chez d'autres architectes de l'époque (Balaÿ, 2003), mais toujours à l'échelle de l'habitation, devrait aussi intéresser nos aménageurs d'aujourd'hui. L'espace intermédiaire entre la sphère publique et la sphère privée, qu'on nomme encore parfois l'entre-deux, ce n'est pas seulement l'espace qui assure une fonction intermédiaire de passage et de transition. Ce peut être un espace particulier, fédérateur de sensations et accompagnateur d'usages.

Mettre l'espace intermédiaire au service de l'accessibilité au logement et à la ville ne doit pas se faire aux dépens des dimensions sensorielles, notamment sonores, comme on l'a vu chez Fourier et Le Corbusier. L'habitant peut souhaiter «engager» la dimension sonore de la ville dans son espace privatif, pour travailler ou se détendre, pour recevoir des visiteurs ou encore pour masquer les bruits domestiques qui l'empêche de se concentrer. Cette gestion directe et instantanée de l'ambiance dans laquelle il souhaite se ressourcer, recevoir ou travailler chez lui, constitue ce qui fera demain la qualité sonore d'une façade d'habitat. La logique de l'isolation phonique n'est donc pas suffisante. Le concepteur d'aujourd'hui a là l'occasion de réfléchir à des dispositifs favorisant l'introduction, par l'habitant, de plusieurs des dimensions temporelles de la réalité sonore urbaine locale (Amphoux 1990, Balaÿ 1994).

Enfin, dernière piste, la culture d'aménageur devrait changer. L'ouïe permet d'envisager des dimensions spatiales affectives et émouvantes au moins aussi riches que celles données par la vue. Lui porter attention, c'est anticiper sur la production d'espaces sonores compatibles avec la fonction sociale pour laquelle ils sont imaginés. Aujourd'hui, au nom de l'hygiène, de la police, d'une représentation sociale commune sur l'espace, beaucoup de propositions spatiales mènent à l'isolement alors 
qu'elles ont été envisagées pour être des lieux de société. Aussi, après l'instauration effective et réglementaire d'une acoustique " scientifique » appliquée à la construction, l'aménagement sonore n'exige-t-il pas un recours singulier et incontournable à des dispositifs éprouvés et à des formes qui plaisent à l'oreille? Il ne s'agit pas de revenir aux modèles sonores du $\mathrm{XIX}^{\mathrm{e}}$ mais de revaloriser une approche intuitive et qualitative de la ville sonante, en aller retour et en confrontations avec le terrain sonore réel, le vécu, l'approche quantitative. $\mathrm{Si}$, dans les décennies récentes, on a voulu, au nom de l'urgence, résoudre un certain nombre de problèmes sonores par des actions d'isolation acoustique fortes et brutales (pour des problèmes de voisinages, de transport...), le contexte actuel évolue rapidement. Il conduit à concevoir un aménagement urbain dont les bases de départ sont très différentes. Les techniques sont bien maîtrisées. Les réglementations sont théoriquement efficaces. Il est temps de commencer une acoustique créative. L'occasion est à saisir. Il va falloir mettre en œuvre un urbanisme sonore qui ne soit pas fonction des seules procédures d'exception, mais qui soit en correspondance avec le développement de l'homme dans le cadre urbain.

Olivier Balaÿ est Architecte et urbaniste à Lyon, enseignant des écoles d'architecture, chercheur au CRESSON (Centre de recherche sur l'espace sonore et l'environnement urbain), UMR CNRS 1563 «Ambiances architecturales et urbaines», Ecole d'Architecture de Grenoble, 60 avenue de Constantine, BP 2636, 38036 Grenoble cedex 2. Olivier.balay@grenoble.archi.fr

\section{Résumé de l'article.}

Aujourd'hui, les bâtisseurs s'interrogent sur le paysage sonore de la ville, sur la sensibilité sociale aux phénomènes audibles et sur les rapports à faire renaître entre l'acoustique, l'architecture et l'urbanisme. Or cette attention portée à la qualité de l'audible n'est pas nouvelle. Les visionnaires du début de l'ère urbaine industrielle avaient déjà imaginé de nouveaux univers sonores, donnant leurs sources d'inspiration, leurs critères de confort acoustique, disant aussi leur croyance en la puissance de récollection par les sons. Trois textes nous ont permis de découvrir tous ces aspects : un texte de Louis Sébastien Mercier - un rêve plutôt - sur Montmartre en l'an 2440 tel qu'il l'imaginait en l'an VII (1798), un texte de C.N.Ledoux sur les lieux en partie construits d'Arc-et-Senans (1804), enfin, dans un texte de 1826, on verra comment Charles Fourier présentait l'organisation sonore de son Phalanstère à partir de l'observation critique des phénomènes sonores de son époque. 


\section{Bibliographie.}

AMPHOUX P. (1990) « Evolution des modes de vie et prospectives de l'habiter sonore $\gg$ in revue Habitation $N^{\circ} 12$, Lausanne, p. 6-11.

ASTIER L., BARON P. (1935), Bruits produits par les distributions urbaines d'énergie électrique et bruits dus à la circulation dans les rues, Paris, Le génie civil.

AUGOYARD J.F. (1978), Les pratiques d'habiter à travers les phénomènes sonores, Chapitre 3 «Contribution à une critique de l'habitat», Plan Construction, Recherche 71.61.375, Grenoble, CRESSON.

BALAŸ O., CHELKOFF G. (1985), La dimension sonore d'un quartier: la cité Mistral à Grenoble, Recherche Plan Construction, Grenoble, CRESSON.

BALAŸ O. (1994), La conception sonore des espaces habités, Recherche MELTE ${ }^{\circ}$ 0592-179, Grenoble, CRESSON.

BALAŸ O. (2003), L'espace sonore de la ville au XIXème siècle, Grenoble, A la croisée.

BACZKO B. (1978), Lumière de l'utopie, Paris, Payot, p. 320.

BERTHELET de BARBOT (1773), Topographie médicale de Lyon et de son territoire, Lyon, p. 5.

CHEVALIER L. (1958), Classes laborieuses et classes dangereuses à Paris pendant la première moitié du XIXe siècle, Paris, Plon.

CHOAY F. (1965), L'urbanisme, utopies et réalités, une anthologie, Paris, Seuil, collection Point Sciences humaines, p. 283.

CHOAY F. (1980), La règle et le modèle. Sur la théorie de l'architecture et de l'urbanisme, Paris, Seuil, collection Espacements, p. 20.

CRUBELLIER M. (1983), « Les citadins et leurs cultures » in DUBY G. (sous la direction de) Histoire de la France urbaine, tome 4 : La ville de l'âge industriel, Paris, Seuil, p. 360 et suivantes.

DELEUZE G. (1973), Empirisme et subjectivité, Paris, PUF, p. 148. 
DUHAMEL G. (1930), Querelles de famille, Paris, Mercure de France.

FARGE A. (1979), Vivre dans la rue à Paris au XVIII e siècle, Paris, Gallimard Archives, p. 36.

FOURIER C. (1829), Le nouveau monde industriel et sociétaire, in CEuvres complètes, Paris, Librairie sociétaire, deuxième édition 1845. Pour les références exactes des citations, cf. Balaÿ O. (2003).

JOSSERAND P., AZAIS C. (1982), «Acoustique et mesure du bruit», in L'oreille oubliée, Paris, Centre Georges Pompidou, CCI, p. 30.

Le CORBUSIER (1946), Manière de penser l'urbanisme, Paris, Architecture d'aujourd'hui, deuxième édition 1963, Paris, Gonthier, p. 7.

Le CORBUSIER (1946-1952), Euvres complètes, Zurich, W. Bösiger, Girsberger, tome 3, p. 194.

Le CORBUSIER (1923), Vers une architecture, Crès, deuxième édition Vincent Fréal, 1958.

LEDOUX C.N. (1804), De l'architecture considérée sous le rapport de l'Art, des Mours, et de la Législation, Paris, Perronneau. Pour les références exactes des citations, cf. Balaÿ O. (2003).

LEQUIN Y. (1983), «Les citadins et leur vie quotidienne» in DUBY G. (sous la direction de) Histoire de la France urbaine, tome 4, Paris, Seuil, p. 354, 379 et 383.

MERCIER L.S. (1798), L'an 2440, Paris. On trouvera un exemplaire des deux volumes à la Bibliothèque de la Sorbonne, Paris, cote R. 427. Pour les références exactes des citations, cf. Balaÿ O. (2003).

OZOUF M. (1984), L'école de la France Paris, Gallimard, Bibliothèque des histoires, p. 319 et 269.

PETITFILS J.C. (1982), La vie quotidienne des communautés utopistes au XIXe siècle, Paris, Hachette, p. 189-190 et p. 259-260. 
PLINE LE JEUNE (97-114) Lettres de Pline Le Jeune, Les belles lettres, Traduction de Guillemin A.M. (1982), in : La Laurentine et l'invention de la villa romaine, Paris, Moniteur, Institut Français d'Architecture (IFA).

ROUSSEAU J.J. (1762), Émile, Paris, E.P. Richard, Éditions Garnier, livre 5, p. 444.

ZELDIN T. (1979), Histoire des passions françaises, Tome 4 " Colère et Politique", Paris, Encres, p. 88. 